\title{
End Relative to Reference Period
}

National Cancer Institute

\section{Source}

National Cancer Institute. End Relative to Reference Period. NCI Thesaurus. Code C82557.

An indication or description that the end of the observation is before, during or after a reference time interval. 